\section{A E E T}

ASOCIACIÓN ESPAÑOLA DE ECOLOGÍA TERRESTRE

\title{
Impactos del nitrógeno agrícola en los ecosistemas acuáticos
}

\author{
A. R. Eugercios Silva ${ }^{1, *}$, M. Álvarez-Cobelas ${ }^{1}$, E. Montero González²
}

(1) Departamento de Biogeoquímica y Ecología Microbiana, Museo Nacional de Ciencias Naturales, CSIC, c/ Serrano, 115 dpdo, 28006, Madrid, España. (2) Departamento de Geodinámica, Facultad de Ciencias Geológicas, c/ José Antonio Novais, 2, Universidad Complutense de Madrid, Ciudad Universitaria, 28040 Madrid, España.

* Autor de correspondencia: A.R. Eugercios Silva [anarolindes@hotmail.com]

> Recibido el 17 de octubre de 2016 - Aceptado el 08 de marzo de 2017

\begin{abstract}
Eugercios Silva, A.R., Álvarez-Cobelas, M., Montero González, E. 2017. Impactos del nitrógeno agrícola en los ecosistemas acuáticos. Ecosistemas 26(1): 37-44. Doi.: 10.7818/ECOS.2017.26-1.06

El desarrollo de la actividad agrícola intensiva de regadío en la segunda mitad del siglo XX, en España pero también en Europa y a nivel global, ha producido que las aguas superficiales y subterráneas se hayan visto sometidas a diversos tipos de estrés. Uno de los más importantes es su estado cualitativo, en el que la lixiviación del exceso de fertilizantes nitrogenados aplicados a los campos agrícolas es el principal mecanismo responsable de las elevadas concentraciones de nitratos medidas en las aguas subterráneas, superiores al límite máximo permitido y recomendado por la legislación existente. Los elevados tiempos de tránsito que normalmente presentan los sistemas acuíferos y su baja capacidad para atenuar la contaminación hacen que éstos se comporten como un almacén de nitrógeno. La exportación del mismo a través del drenaje superficial por su conexión hídrica con cuerpos de agua superficiales constituye el principal mecanismo de contaminación por nitratos de ríos, lagos, mares y océanos. Esas elevadas concentraciones producen problemas de tipo ambiental, como la progresiva eutrofización de sus aguas, así como riesgos para la salud humana, como la enfermedad del "bebe azul". Para lograr la recuperación de los sistemas y alcanzar el buen estado ecológico de las masas de agua superficiales y subterráneas, se hace necesario el estudio exhaustivo de su funcionamiento hídrico y del origen, el destino y los procesos de transformación a los que es sometido el nitrógeno.
\end{abstract}

Palabras clave: nitratos; agua subterránea; recursos hídricos; eutrofización; ciclo biogeoquímico del nitrógeno

Eugercios Silva, A.R., Álvarez-Cobelas, M., Montero González, E. 2017. Agricultural nitrogen impacts on aquatic ecosystems. Ecosistemas 26(1): 37-44. Doi.: 10.7818/ECOS.2017.26-1.06

Groundwater and surface water bodies have been affected to various stress types since the second half of the twentieth century due to the development of intensive agricultural irrigation activities not only in Spain but also in Europe and globally. Leaching of nitrogen from fertilizers applied to agricultural fields affects the qualitative state of groundwater resources, due to the high nitrate concentrations measured in this water bodies, above the maximum allowable limit. Groundwater acts as a long-term sink for nitrate due to the high transit times and low remediation capacity to mitigate pollution. Nitrogen exportation through the discharge to surface water bodies is the main mechanism of nitrate pollution of rivers, lakes, seas and oceans. High nitrate concentrations cause environmental issues such as the progressive water eutrophication and human health risks ("blue baby" disease). To achieve good ecological status of water bodies is necessary the comprehensive study of the conceptual model, taking into account the hydric behavior and the source, fate and its transformation processes of the nitrogen cycle.

Key words: nitrate; groundwater; water resources; eutrophication; nitrogen cycle

\section{Introducción}

El ciclo biogeoquímico del nitrógeno es uno de los más importantes de la biosfera (Galloway et al. 2003). Para su estudio, es necesario tener en cuenta un vasto conjunto de procesos físicos, químicos y biológicos a los que se ve sometido en su recorrido por los diferentes sistemas terrestres. Los procesos principales de transformación de nitrógeno son esencialmente cinco: fijación, asimilación, amonificación, nitrificación y desnitrificación, aunque otros procesos involucrados en el ciclo global del nitrógeno son la volatilización del amoniaco y la lixiviación de nitrógeno hacia el agua subterránea (Fig. 1). La acción humana ha causado un enorme impacto en el ciclo global del nitrógeno, produciendo la entrada de $\mathrm{Nr}$ antropogénico en el sistema terrestre y generando una serie de impactos ambientales, aunque la aplicación de fertilizantes en las áreas agrícolas es el que cobra mayor importancia.
La intervención del ser humano en los diferentes ecosistemas ha provocado una alteración del ritmo natural del ciclo del nitrógeno, lo que conlleva una serie de consecuencias ambientales. Las actividades humanas como la adición excesiva de fertilizantes nítricos y amoniacales en las superficies agrícolas, los vertidos de aguas residuales y fosas sépticas, la lixiviación de purines procedentes de las actividades ganaderas (abonos) o la percolación de lixiviados procedentes de estercoleros o vertederos son las principales causas de los problemas de contaminación por nitrógeno de origen antropogénico (Fig. 1). Además, la emisión de gases en forma de óxidos de nitrógeno procedentes de fábricas, vehículos y quema de combustibles genera problemas asociados a la lluvia ácida y constituye una fuente importante de nitrógeno en los ecosistemas naturales hasta entonces no perturbados, así como la fijación biológica de nitrógeno antropogénico por campos de leguminosas (Tabla 1). 

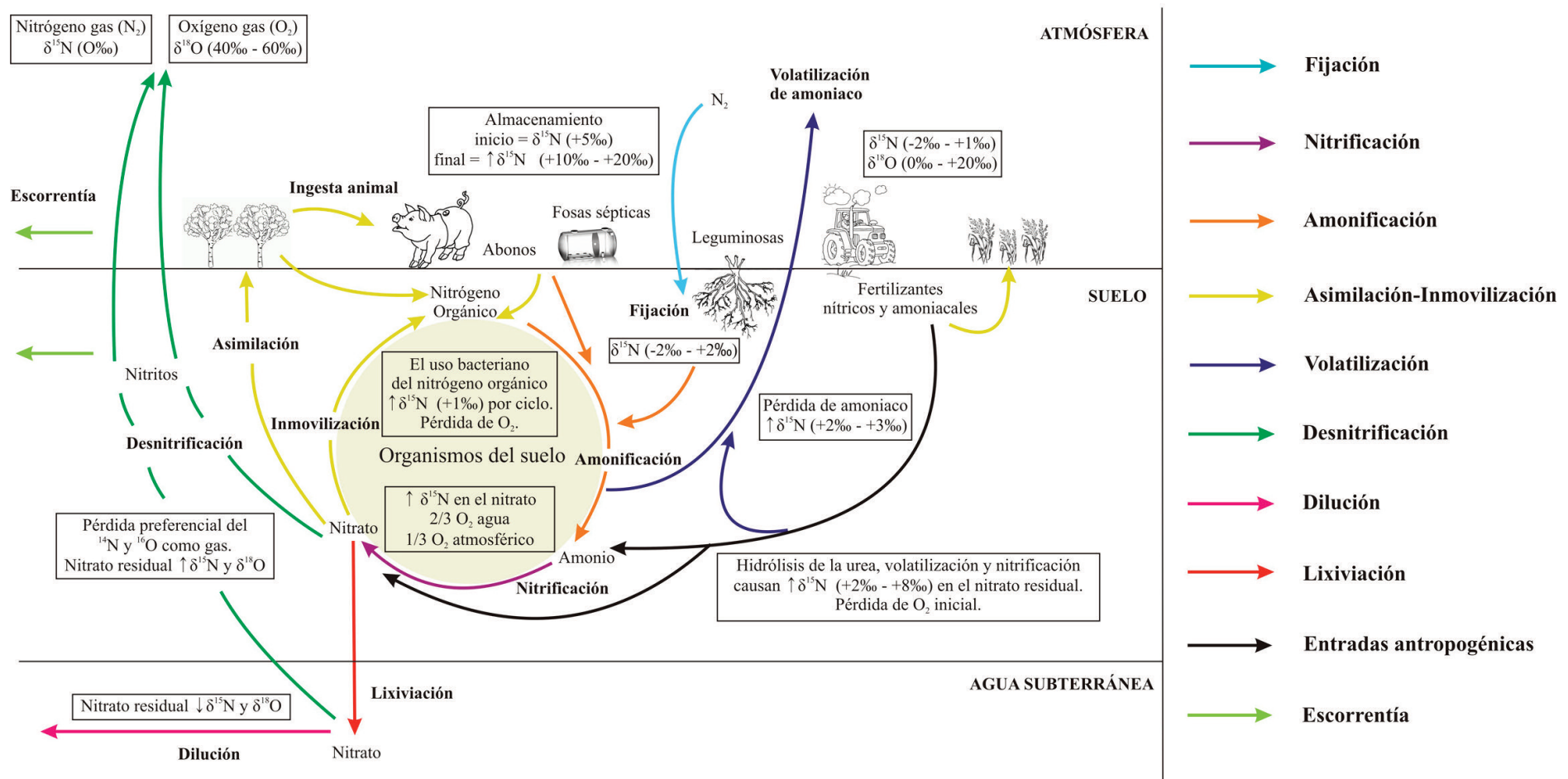

Figura 1. El ciclo del nitrógeno y transformaciones isotópicas del nitrato. Fuente: Eugercios (2013)

Figure 1. The nitrogen cycle and nitrogen isotopic transformations. Source: Eugercios (2013)

Tabla 1. Importancia relativa de las fuentes puntuales y difusas de nitrógeno antropogénico que contribuyen a modificar el ciclo natural del nitrógeno a nivel global.

Table 1. Relative importance of anthropogenic nitrogen point sources and diffuse sources that modifies the global nitrogen cycle.

\section{Fuentes difusas}

Lixiviación de fertilizantes en las superficies agrícolas

Emisión de gases de fábricas, vehículos

y quema de combustibles

Fijación biológica de nitrógeno antropogénico

en los campos de leguminosas, arroz, etc.

Lixiviación de purines de actividades ganaderas

\section{Fuentes puntuales}

Percolación de lixiviados de vertederos

Vertidos de aguas residuales

1 Mayor importancia

3 Menor importancia

Del total del $\mathrm{Nr}$ antropogénico introducido en el ciclo terrestre, aproximadamente $150 \mathrm{Tg} \mathrm{N} \mathrm{a}^{-1}$ (teragramos de nitrógeno al año o millones de toneladas métricas al año), $9 \mathrm{Tg} \mathrm{N} \mathrm{a}^{-1}$ permanecen retenidas a largo plazo en los suelos agrícolas. La desnitrificación, proceso natural de transformación de nitrato en nitrógeno molecular gaseoso, es capaz de eliminar $17-20 \mathrm{Tg} \mathrm{N}^{-1}$ de los suelos, mientras que se produce el transporte atmosférico de $48 \mathrm{~T} \mathrm{~N} \mathrm{a}^{-1}$ desde las zonas terrestres hacia el interior de los océanos. Sin embargo, la mayor parte del $\mathrm{Nr}$ antropogénico $\left(65 \mathrm{Tg} \mathrm{N} \mathrm{a}^{-1}\right)$ es transferida hacia los ecosistemas acuáticos, especialmente mediante contaminación difusa desde los campos agrícolas; $15 \mathrm{Tg} \mathrm{N}^{-1}$ son lixiviadas hacia el agua subterránea, permaneciendo retenidas, y 35-50 $\mathrm{Tg} \mathrm{N} \mathrm{a}^{-1}$ son exportadas a través de los ríos y juegan un papel crucial en el suministro de nutrientes a los océanos (Schlesinger
2009). Parte de ese nitrógeno exportado a través de los ríos es retenido en los lagos, especialmente en los más pequeños $\left(<50 \mathrm{~km}^{2}\right)$, en los que 9.3 $\mathrm{Tg} \mathrm{N}^{-1}$ son eliminadas por desnitrificación y sedimentación (Harrison et al. 2008). El hecho de que la acción humana haya doblado las entradas de $\mathrm{Nr}$ en el ciclo global del nitrógeno provoca su acumulación en todos los reservorios terrestres (atmósfera, suelo y agua), lo que conlleva consecuencias negativas, tanto para el medio ambiente como para la salud humana. Incluso, produce la alteración de los ciclos globales de otros elementos químicos como el fósforo, el sulfuro y el carbono (Gruber y Galloway 2008). Estas implicaciones son lo que se conoce como "la cascada del nitrógeno" (Galloway et al. 2003).

La contaminación de los recursos hídricos por nitrógeno se puede producir por fuentes de tipo puntual o difusa. En los casos de fuente de tipo puntual, la contaminación se produce en un área localizada y restringida, tratándose normalmente de vertidos residuales o efluentes, en los que el usuario causante del daño normalmente está identificado. La contaminación difusa se produce por la lixiviación de nitrógeno desde superficie hacia los cuerpos de agua subterráneos y superficiales a través de un área muy amplia, en el que se identifican un gran número de usuarios como causantes de la misma. Así, la agricultura es una de las principales fuentes de contaminación difusa de los recursos hídricos por nitrógeno y fósforo (Almasri 2007; Cherry et al. 2008).Además, generan otros muchos impactos asociados a nivel mundial (FAO 2011), como la escasez de agua (Asia y América del Norte), inundaciones (Asia y África), pérdida de biodiversidad (Asia), deforestación (América del Sur y África), desertificación (África), pérdida de fertilidad en los suelos (África), procesos erosivos (África y Asia) y escasez de terrenos cultivables (Asia y África). (Tabla 2). En Europa, y más específicamente en España, los problemas más importantes relaciones con la agricultura son la escasez de agua, pérdida de biodiversidad y la contaminación de los recursos hídricos. Así, el creciente desarrollo de la agricultura durante las últimas décadas ha provocado que este tipo de contaminación difusa represente un problema muy extendido en todo el mundo (Strebel et al. 1989; FAO 2011) y desde hace tiempo, se reconoce como un importante problema ambiental, documentado ampliamente en la literatura científica. 
Tabla 2. Importancia relativa de los riesgos asociados a la agricultura para cada región geográfica.

Table 2. Relative importance of the risk associated to agricultural activities for each global region.

América del Norte América del Sur Centroamérica Europa España Asia África Oceanía

\begin{tabular}{|c|c|c|c|c|c|c|c|c|}
\hline Inundaciones/aumento del nivel del mar & 2 & 2 & 1 & 3 & - & 1 & 1 & 2 \\
\hline Escasez de agua & 1 & - & - & - & 1 & 1 & - & - \\
\hline Contaminación & 1 & - & - & 1 & 1 & 1 & - & - \\
\hline Pérdida de biodiversidad & 3 & 2 & - & - & 1 & 1 & 3 & 2 \\
\hline Deforestación & - & 1 & 2 & - & - & - & 1 & 2 \\
\hline Desertización/sequía & - & - & - & - & - & 1 & 1 & - \\
\hline Baja fertilidad del suelo & - & 2 & 2 & - & - & - & 1 & - \\
\hline Erosión & 3 & 2 & 2 & - & - & 1 & 1 & - \\
\hline Escasez de terreno disponible & - & - & 2 & - & - & 1 & 1 & 2 \\
\hline
\end{tabular}

1 Mayor importancia.

3 Menor importancia

\section{El ciclo agrícola}

En las áreas agrícolas, la lixiviación de nitrógeno disuelto, tanto por precipitación como por riego, está controlada por el ciclo del cultivo. Los fertilizantes más usados son de tipo ureico, amoniacales y otros fertilizantes orgánicos aplicados en sementera. Los fertilizantes de tipo nítrico se aplican en cobertera, al inicio del crecimiento del cultivo, momento de mayor demanda de nutrientes por las plantas. Además, se utilizan fertilizantes ternarios NPK, compuestos por los tres macronutrientes principales (nitrógeno, fósforo y potasio) para su aplicación en sementera y cobertera. Desde que se produce la fertilización del terreno hasta el momento de recogida de la cosecha, el agua de lluvia y el riego continuado provocan la disolución del fertilizante presente en el suelo, que es absorbido por las raíces de las plantas. Eugercios (2013) estudió la lixiviación de nitrógeno en las parcelas agrícolas hacia el agua subterránea del acuífero de Campo de Montiel (Castilla-La Mancha) y obtuvo que es mínima durante todo el ciclo de crecimiento vegetativo (abril a julio), dada la elevada evapotranspiración potencial durante estos meses y la toma de nutrientes por las plantas (Fig. 2). Después de la recogida de la cosecha, en los meses estivales, el suelo queda yermo, sin cobertera vegetal. El inicio de la época de lluvias en otoño provoca la disolución del fertilizante ex- cedentario que permanece aún en el terreno. La ausencia de cobertera vegetal, la elevada precipitación y la baja evapotranspiración potencial en estos meses provoca la lixiviación masiva de nitrato en el último trimestre del año.

La cantidad total de nitrógeno lixiviado depende del tipo de cultivo, de la dinámica del nitrógeno en el suelo, de las prácticas agrícolas, de las características del perfil del suelo, de la presencia de materia orgánica en el suelo, de las características pluviométricas del año simulado (año húmedo o seco) y de la zona climática (Haygarth et al. 2003). Este valor se estima entre un 5\% y un $25 \%$ del total de fertilizante aplicado, aunque otros autores citen coeficientes superiores, desde 30 a 50\% (Goderya et al. 1996; Shamrukh et al. 2001). Los coeficientes de lixiviación de nitrógeno calculados en otras zonas de España muestran valores similares: Eugercios (2013) estima que entre el $11-34 \%$ del fertilizante aplicado anualmente es lixiviado hacia el acuífero del Campo de Montiel; DíazLezcano (2007) calcula unas tasas de lixiviación en los acuíferos de la cuenca del río Henares comprendidas entre 0.34 y $10 \mathrm{mg} \mathrm{N}$ $\mathrm{m}^{-2} \mathrm{~d}^{-1}$ (miligramos de nitrógeno por metro cuadrado al día), que corresponden al $20-35 \%$ del fertilizante aplicado en cultivos de cereales de invierno, maíz, y forrajeras. Todos estos autores subrayan la importancia de la precipitación en la lixiviación de nitrógeno, en mayor o menor medida.
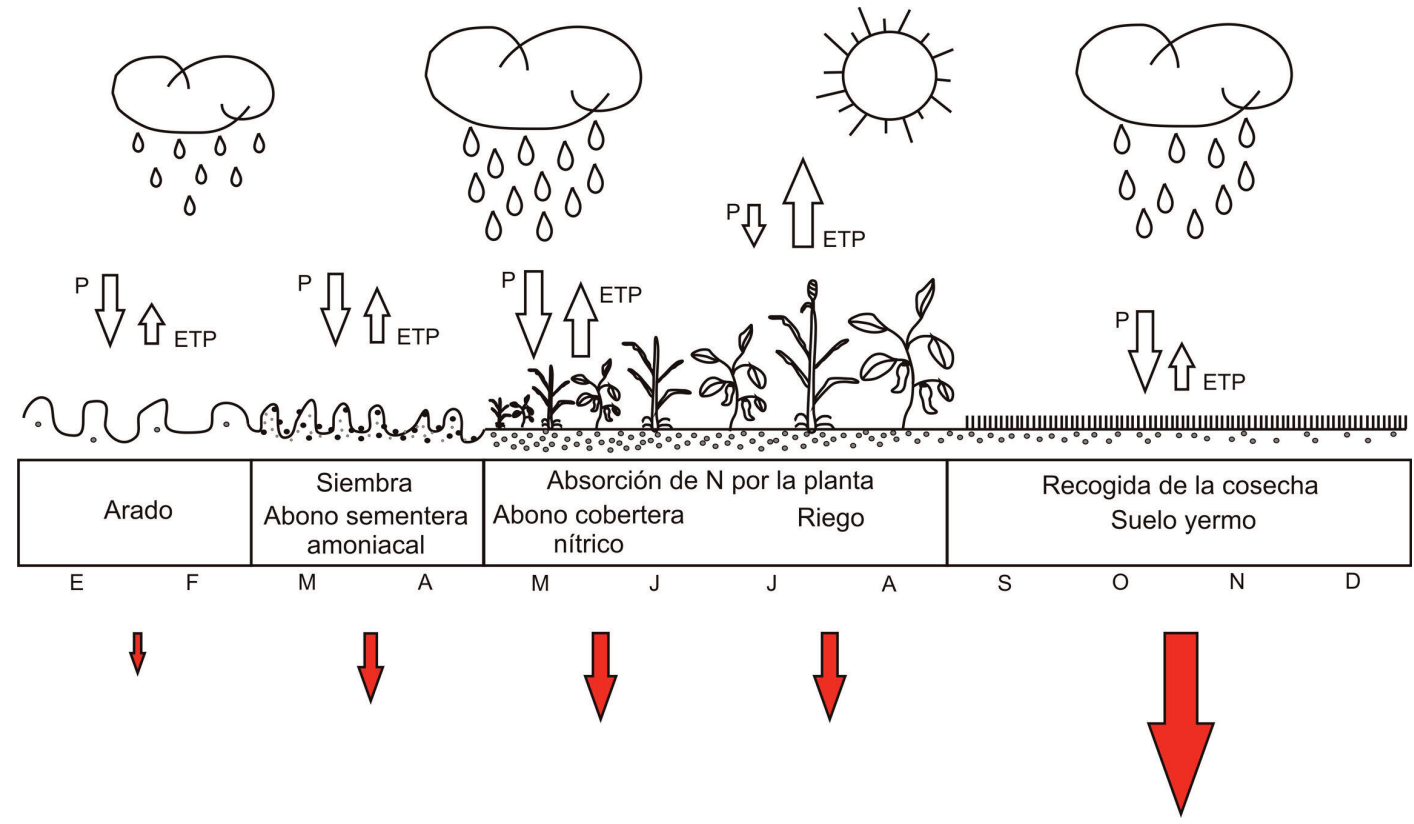

Figura 2. Lixiviación de nitrógeno (flechas rojas) en el área agrícola del Campo de Montiel (P, precipitación; ETP, evapotranspiración potencial). El tamaño de las flechas indica la cantidad de nitrógeno lixiviado. Fuente: Eugercios (2013).

Figure 2. Nitrogen lixiviation (red arrows) in agricultural área of Campo de Montiel (P, rainfall; ETP, evapotranspiration). Arrow size means the amount of nitrogen leached. Source: Eugercios (2013). 


\section{Impactos en el agua subterránea}

La lixiviación de nitrógeno desde las parcelas agrícolas es la principal responsable de la contaminación por nitratos de las aguas subterráneas. Con respecto al impacto en la Huella Hídrica de los cultivos, la lixiviación por nitrógeno se reconoce como el principal compuesto asociado a la cuantificación de la Huella Hídrica Gris (Chenoweth et al. 2014). En España, representa un problema muy importante acrecentado en las últimas décadas, especialmente en regiones con grandes superficies agrícolas como en la costa mediterránea, las cuencas del Ebro, Guadiana y del Guadalquivir y las regiones interiores de Castilla-La Mancha (Varela 1991). En la Figura 3 , se muestra un mapa con la concentración máxima de nitratos medida en alguno de los puntos de la red de control de cada masa de agua subterránea. Aproximadamente, el $25 \%$ de las masas de agua subterránea existentes están afectadas por la contaminación difusa por nitratos (Fig.3), en el que la lixiviación de nitratos por la aplicación de fertilizantes nitrogenados en las parcelas agrícolas es la causa principal de esta situación. Quizá represente un problema difícil de abordar, dada la aplicación cada vez más excesiva de fertilizantes en estos terrenos agrícolas, las elevadas tasas de renovación del agua subterránea que los caracterizan como amplios almacenes de nitrógeno y la distribución de competencias de cuencas entre varios organismos, que quizá pueden hacer más difícil su gestión. Así, en las provincias de Jaén y Granada en la región Subbética se ha detectado contaminación por nitratos de origen agrícola en más de un tercio de los manantiales y acuíferos de esta región en los últimos años 2014-2016 (EEA 2016). Igualmente, en la Confederación Hidrográfica del Ebro ( $\mathrm{CHE}$ ) se han detectado 20 zonas acuíferas (36 masas de agua subterránea) afectadas por contaminación por nitratos de origen agrícola, justo en las zonas con mayor población de abastecimiento urbano (CHE 2016). En la región de Baleares, el $27 \%$ de las masas de agua contienen elevadas concentraciones de nitratos de origen agrícola (PHIB 2015).
Se reconoce como uno de los principales problemas ambientales, en el que la principal consecuencia es la disminución de la calidad del agua y problemas de acidificación (Camargo y Alonso 2007). Este incremento de nutrientes en los ecosistemas acuáticos produce un aumento de la producción primaria (fotosíntesis), un incremento masivo de algas, con un deterioro posterior de la estructura, composición y dinámica del sistema. Este incremento algar se traduce en un aumento de la turbidez, aumento de la actividad de las bacterias descomponedoras, disminución de oxígeno y muerte masiva de organismos existentes, resultando todo ello en una degradación progresiva del hábitat. A su vez, también es causante de impactos en la salud humana como la enfermedad conocida como metahemoglobinemia o "síndrome del bebé azul", ya que afecta principalmente a infantes (Townsend et al. 2003). El consumo de agua con elevadas concentraciones de nitrato genera un aumento de metahemoglobina en sangre, reduce la concentración de oxígeno y produce dificultad para respirar, fatiga, cianosis, pérdida de conocimiento e incluso la muerte. Otras enfermedades, como el cáncer o problemas digestivos y reproductivos, se han asociado al consumo de agua contaminada por nitratos, pero actualmente no existe un consenso sobre el tema, por lo que continúa siendo objeto de amplios debates en la comunidad científica.

La Unión Europea dictó dos directivas para la protección de las aguas de la contaminación por nitrato de origen agrícola. En primer lugar, la Directiva de Nitratos (91/676/CEE), traspuesta a la legislación española por el Real Decreto 261/1996, establece un umbral de concentración por nitrato de $50 \mathrm{mg} / \mathrm{l}$ para la designación de aguas contaminadas, y su objetivo principal era la reducción de la contaminación por nitrato de origen agrario y la designación de Zonas Vulnerables a la contaminación por nitrato. Por otra parte, la Directiva Marco del Agua (DMA 2000/60/CE) del Parlamento Europeo establece un marco comunitario de actuación en el ámbito de la política de aguas, que insta a los Estados Miembros para que sus aguas (continentales, subterráneas, costeras y de transición) presentaran un buen estado ecológico para el año 2015, o en su defecto para los horizontes 2021 o 2027.

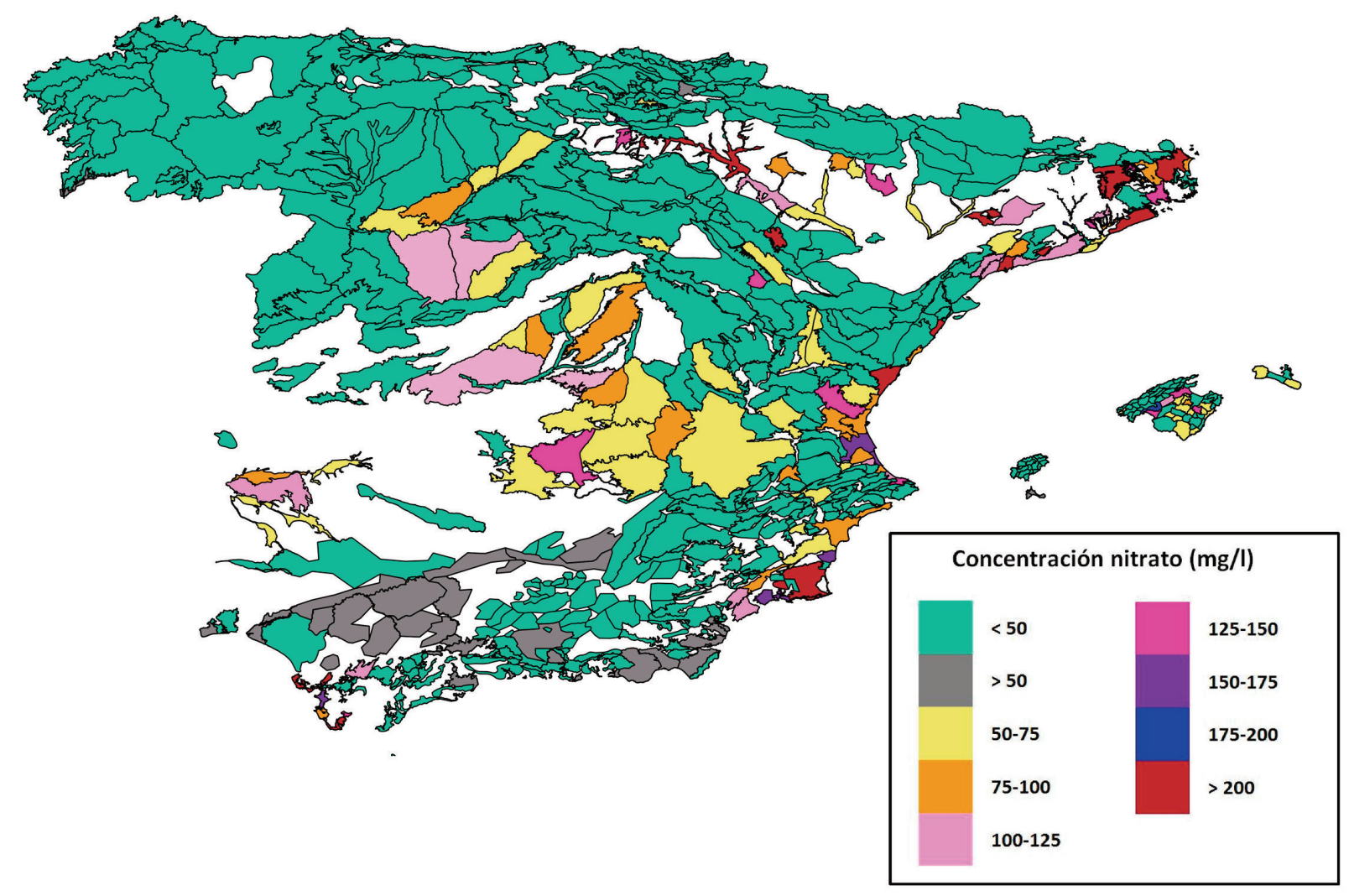

Figura 3. Masas de agua subterránea contaminadas por nitratos (2015). Fuentes: ACA (2015); CHD (2015); CHE (2016); CHG (2015); CHGuad. (2015); CHJ (2015); CHS (2016); CHT (2015); JDA (2015); PHIB (2015).

Figure 3. Groundwater bodies contaminated by nitrates (2015). Sources: ACA (2015); CHD (2015); CHE (2016); CHG (2015); CHGuad. (2015); CHJ (2015); CHS (2016); CHT (2015); JDA (2015); PHIB (2015). 
Cherry et al. (2008) hicieron una revisión de los métodos existentes para minimizar la pérdida de nutrientes procedentes de la agricultura y llegan a la conclusión de que es improbable que, de forma general, las masas de agua, esencialmente las subterráneas, lleguen al buen estado ecológico requerido en tan poco tiempo, independientemente del método utilizado, debido a los elevados tiempos de tránsito que presentan. Por este mismo motivo, Holden et al. (2015) destacan la conveniencia y necesidad de considerar escalas temporales de varias décadas en la gestión de nutrientes. De ahí surge la necesidad de estudiar diferentes aspectos relacionados con la contaminación de tipo no puntual, como su origen, su transporte y su destino en cuencas forestales, agrícolas y urbanas (Kaushal et al. 2011), definirlas y caracterizarlas (Gang et al. 2005) o analizar los principales enfoques teóricos y económicos existentes para la regulación de dichas fuentes contaminantes (Xepapadeas 2011). Pese a la existencia de procesos naturales de eliminación de nitrato (desnitrificación) y otros métodos tecnológicos más complicados y costosos, la prevención dentro de un marco de gestión de análisis de decisión multicriterio continúa siendo la mejor herramienta para evitar la lixiviación de nitrógeno. El empleo de códigos de buenas prácticas agrarias, entre los cuales hay que considerar los cambios de los métodos de cultivo, las tasas fiscales, los límites máximos de aplicación de fertilizantes, la conveniencia de rotación de los cultivos, el uso de sistemas eficientes de riego y la difusión de campañas de asesoramiento, disminuiría los efectos negativos de la agricultura en la calidad química del agua subterránea (Almasri 2007).

Estas medidas de prevención deberían constituir una prioridad en aquellos países menos contaminados. Por ejemplo, Schindler et al. (2006) vieron que la contaminación por nitratos de las aguas superficiales y subterráneas de Canadá comienza a representar un problema debido a la rápida expansión de la población humana, de la industria y la agricultura y alertan de la necesidad de ponerle freno antes de que la situación sea irremediable. Quizá sea demasiado tarde para algunas zonas de Europa. Tal y como aseguran O Shea y Wade (2009), aunque la política fiscal y la gestión pueden dar lugar a cambios significativos en el uso de la tierra y a la disminución de fertilizantes nitrogenados aplicados, la magnitud de estos cambios no se verían reflejados en una reducción de la concentración de nitrato en el agua superficial y subterránea, debido al enorme stock de nitrato presente en las zonas saturadas y no saturadas. Otro ejemplo en España ocurre en el acuífero del Campo de Montiel, en el que la contaminación por nitratos permanecerá en el acuífero durante varias décadas, aunque se suprimiera por completo la adición de fertilizantes en el área, debido a los elevados tiempos de tránsito del agua subterránea (Eugercios 2013). De aquí surge la necesidad de un profundo estudio sobre el funcionamiento y la evolución de los acuíferos, su interacción con las aguas superficiales y las afecciones que pueden repercutir en el sistema fluvio-lacustre, tanto desde el punto de vista de la cantidad como de la calidad. Tal y como ya pusieron de manifiesto Turner et al. (2006), Galloway et al. (2008) y Holden et al. (2015) es necesaria la cooperación interdisciplinaria de científicos de diferentes áreas (hidrología, hidrogeología, hidrología isotópica, microbiología, etc) y un análisis multicriterio para abordar y mejorar la comprensión del destino y el transporte de los contaminantes difusos en las cuencas agrícolas.

\section{Impactos en el agua superficial de arroyos, lagos y humedales}

La contaminación por nitratos no sólo afecta al agua subterránea sino también a la superficial, ya que ambas suelen estar conectadas y forman un único sistema (Winter 1999). Entre los numerosos procesos que forman parte del ciclo del nitrógeno, uno de los más importantes es el estado hidrológico e hidrogeológico del medio estudiado, que determina la conexión hídrica entre las aguas subterráneas y las superficiales. Por ejemplo, aunque se restringiera la aplicación de fertilizantes en el área en los próximos años, la exportación de nitrógeno hacia ríos, lagos y océanos continuaría siendo de gran importancia al recibir el nitrógeno almace- nado en el agua subterránea. Kennedy et al. (2009) encontraron una relación inversa entre la concentración de nitrato y la edad del agua subterránea que descargaba en un arroyo de Carolina del Norte; calcularon un tiempo de entre 20-30 años entre la entrada de nitrato hacia el agua subterránea procedente de las actividades agrícolas y la salida del mismo mediante su descarga hacia el arroyo. Si los acuíferos representan almacenes temporales de nitrato con elevados tiempos de tránsito y gran parte de las aguas superficiales están alimentadas por agua de origen subterráneo, en un futuro la principal forma de nitrógeno exportado sería el nitrato procedente de las actividades agrarias. Queda patente la repercusión que esto tiene en la gestión de la calidad del agua en una cuenca. Tal y como sugieren Álvarez-Cobelas et al. (2008), lo más adecuado sería un enfoque del tipo caso a caso, basado en estudios locales o regionales detallados para predecir la exportación de nitrógeno global.

Con respecto a la exportación de nitrógeno hacia arroyos y lagos, en varias cuencas semiáridas del mundo representa aproximadamente el $15 \%$ de las entradas totales de nitrógeno, como en la cuenca baja del río Oglio de la llanura del río Po al norte de Italia (Soana et al. 2011), en el acuífero aluvial del valle del río Mississippi (Welch et al. 2011) y en otras partes del mundo (Álvarez-Cobelas et al. 2008). Belanger et al. (1985) calcularon que el 17.6 y el $8.7 \%$ de las entradas totales de nitrógeno y fósforo en el lago East Tohopekaliga procedían de entradas difusas de agua subterránea del acuífero kárstico de Florida. En España, la exportación de nitrógeno en el acuífero del Campo de Montiel hacia las Lagunas de Ruidera constituye otro mecanismo importante de eliminación de nitrógeno, equivalente al 22-23\% del fertilizante aplicado (Eugercios 2013).

La desnitrificación se considera la principal vía de eliminación de nitrógeno en lagos, ríos y humedales. Es del orden de $35 \mathrm{Tg} \mathrm{N}$ $\mathrm{a}^{-1}$ en ríos, $31 \mathrm{Tg} \mathrm{N} \mathrm{a}^{-1}$ en lagos y $8 \mathrm{Tg} \mathrm{N}^{-1}$ en estuarios, en el que en todos los casos, las entradas totales de $\mathrm{Nr}$ y el tiempo de residencia del agua ejercen como los factores principales de control de la desnitrificación (Seitzinger et al. 2006). Groffman et al. (2009) afirman que la desnitrificación es un proceso difícil de medir y modelizar, ya que un alto porcentaje de la misma en los sistemas acuáticos ocurre en pequeñas áreas (hotspots) y en periodos breves de tiempo (hot moments). Por ejemplo, en España, en las Lagunas de Ruidera (laguna Conceja) de Castilla-La Mancha, se identificó como momento más favorable (hot moment) para la desnitrificación el verano, durante el que podría ser más elevada en las zonas más someras de la laguna (hotspots) y en las zonas de ribera (Eugercios 2013). Las zonas de interacción entre las aguas subterráneas y superficiales presentan características físico-químicas específicas que afectan a la hidrología, la ecología y a los procesos biogeoquímicos del nitrógeno. Winter et al. (2008) vieron que el agua subterránea presentaba condiciones anóxicas y una composición química diferente al atravesar el cauce de un arroyo e interaccionar con los materiales del lecho. Desde un punto de vista ecológico, la presencia de microalgas bentónicas puede estimular la nitrificación durante el día y la desnitrificación durante la noche (Groffman et al. 2009). Algunas especies de macrófitas acuáticas estimulan la desnitrificación en la rizosfera (Ottosen et al. 1999). Incluso, la distribución de las bacterias desnitrificantes en el agua y en el sedimento varía dependiendo de la estratificación térmica y del estado trófico del lago (Kim et al. 2011).

En el futuro, el nivel crítico de entradas de nitrógeno hacia los sistemas lagunares deberá ser mucho menor para mantener el buen estado ecológico de los lagos mediterráneos (Jeppesen et al. 2011).

\section{Impactos en el agua superficial de mares y océanos}

La exportación de nitrógeno puede alcanzar los sistemas costeros. La exportación de $35-50 \mathrm{Tg} \mathrm{N} \mathrm{a}^{-1}$ a los océanos, a través de los ríos y de las descargas de agua subterránea, ha provocado problemas de eutrofización, ausencia de oxígeno y degradación de hábitats (Rabalais 2002; Díaz y Rosenberg 2008). Muchas áreas costeras del mundo se encuentran afectadas, 
ocmo las costas de Australia, Europa y Estados Unidos, incluyendo además el mar Báltico y Adriático. Un caso reciente y de especial interés en España es el correspondiente al sistema hidrológico Campo de Cartagena-Mar Menor. El Mar Menor es un ecosistema único incluido en el convenio Ramsar de humedales de importancia internacional y protegido como espacio natural y zona de especial interés. En los últimos años 2014-2016, se ha detectado una progresiva eutrofización de este ecosistema, presentándose problemas de turbidez en 40 playas del Mar Menor, con presencia masiva de fitoplancton que representa un claro síntoma de degeneración. Las causas de esta degradación son múltiples, aunque se destaca una principal consistente en la lixiviación de nitratos hacia el acuífero de los fertilizantes adicionados en las cuencas agrícolas del Campo de Cartagena y su posterior exportación hacia el Mar Menor. Además, la degradación de los humedales existentes previos a la laguna del Mar Menor, que actuaban como filtro de la contaminación, acrecienta aún más el problema existente, en el que su recuperación se reconoce como el método más eficaz para disminuir los nutrientes exportados al Mar Menor (Martínez 2013).

La mayor parte de dicho $\mathrm{Nr}$ se pierde por desnitrificación en las plataformas oceánicas o es atrapado y enterrado en los sedimentos costeros, por lo que no alcanza la zona central de las grandes regiones oceánicas (Seitzinger et al. 2006). Teniendo en cuenta que la mayor parte del $\mathrm{Nr}$ presente en los ríos es desnitrificado en las zonas costeras (Seitzinger et al. 2006; Schlesinger 2009) y devuelto a la fase gaseosa como $\mathrm{N}_{2}$, la exportación de nitrógeno a través de escorrentía superficial quizá represente uno de los métodos más eficaces para la eliminación de nitrógeno de forma global.

La situación futura tampoco es muy alentadora. Seitzinger et al. (2010) modelizaron las exportaciones de N de forma global a través de las cuencas de los ríos hacia las zonas costeras para cuatro futuros escenarios del año 2050, basados en las diferencias en el futuro desarrollo de los ecosistemas y en la evolución política, económica y poblacional. Sus resultados muestran un incremento de la exportación de $\mathrm{N}$ de un $5-10 \%$ para los escenarios menos favorables, mientras se produce un descenso leve (2-3\%) en los escenarios con un enfoque proactivo en la gestión de los ecosistemas. En todos los casos, es la región del sur de Asia (con rápido desarrollo económico e incremento poblacional) la que domina las tendencias globales de exportación de nitrógeno. Esto indica que un total de 42-48 Tg N a ${ }^{-1}$ continuarán exportándose hacia los océanos en el año 2050, si bien existirá una gran variabilidad entre regiones más desarrolladas, como Europa y América del Norte, con respecto a África, América del Sur o el sur de Asia.

\section{Evolución futura}

Debido al aumento progresivo de la población mundial, es más que previsible un incremento de las entradas de $\mathrm{Nr}$ de origen antropogénico en el ciclo terrestre, especialmente a través de la contaminación difusa debida a la aplicación de fertilizantes, producidos mediante el proceso industrial Haber-Bosch, en los campos agrícolas. Bouwman et al. (2009) realizaron balances de nitrógeno en los suelos agrícolas para cuatro futuros escenarios del año 2050 , en el que en todos los casos, se produciría un incremento de $\mathrm{N}$ en los suelos (154-231 $\mathrm{Tg} \mathrm{N} \mathrm{a}^{-1}$ ) y se perdería un $24-30 \%$ por volatilización del amoniaco, un 46-49\% por desnitrificación y un 23-26\% por lixiviación. En dichos balances, la lixiviación de $\mathrm{N}$ hacia los ecosistemas acuáticos representa un total de $36-59 \mathrm{Tg} \mathrm{N} \mathrm{a}^{-1}$, cantidad elevada a pesar de haberse tenido en cuenta una mayor eficiencia en el uso del nitrógeno en todos los escenarios.

En Europa, las pautas futuras del sector agrícola dependen en gran medida de los acuerdos establecidos en el Parlamento Europeo sobre la Política Agraria Comunitaria (PAC), en cuanto a la gestión de las subvenciones que se otorgan a la producción agrícola en la Unión Europea. El cumplimiento de todas las exigencias europeas en un momento de crisis económica hizo necesaria una reforma de la PAC en el año 2013, por lo que se establecían las pautas y nor- mativa a seguir para el ejercicio presupuestario 2014-2020. Entre los retos que se deberían abordar para el futuro se incluían: asegurar una producción de alimentos viable, dada la inestabilidad de los mercados y la crisis de precios; reducir el impacto sobre el medio ambiente mediante la gestión sostenible de los recursos naturales; y tener en cuenta los efectos que el Cambio Climático (CC) pueda tener de forma directa o indirecta sobre la gestión agrícola, sugiriendo planes de adaptabilidad al mismo (PAC 2013). Quizá es demasiado pronto para saber si estas medidas han surtido efecto en la gestión agrícola y sus efectos en los recursos hídricos.

El CC, dentro del Cambio Global, puede considerarse como una de las principales presiones que afectan a los sistemas hidrológicos y a los recursos hídricos (IPCC 2001, 2007 y 2013). EI CC producirá variaciones en la temperatura y en la precipitación en las próximas décadas, lo cual repercutirá no solamente en un punto de vista de la cantidad del recurso (Loaiciga et al. 2000), sino también en la calidad química del agua de todos los sistemas interconectados (Arnell 1998). El CC es especialmente importante en los ecosistemas agrícolas, en los que las variaciones en la precipitación y temperatura provocarán cambios en el ciclo del cultivo, alterando los momentos de siembra y recogida de la cosecha y los ciclos de fertilización (OECC 2006). La disminución de las precipitaciones en primavera y verano, provocará un aumento en la necesidad de riego de los cultivos, adelantándose la época de siembra o prevaleciendo el cultivo de plantas con ciclo corto (Dominguez et al. 2009). Esto producirá un incremento de un $289 \%$ en las tasas de lixiviación de nitrógeno en otoño (Patil et al. 2010), debido a que el suelo permanecerá yermo durante periodos de tiempo mucho mas prolongados. Olesen et al. (2007) estimaron la lixiviación de nitrato, a partir de unas tasas óptimas de fertilización, para el horizonte 2071-2100 utilizando nueve modelos climáticos regionales (RCM) con un escenario de emisiones A2. En el norte de Europa, predomina la disminución en las tasas de lixiviación de nitrato, mientras que en el sur se observa mayor variabilidad. En España, la reducción de la lixiviación por nitrato es extensible a la mitad norte peninsular, mientras que en las cuencas del Ebro, del Guadalquivir y en el tramo final de la cuenca del Guadiana (Portugal) aumentará la lixiviación por nitrato. En el resto de España, la situación es imprecisa y dependerá de las condiciones locales del suelo, de las condiciones climáticas y de los balances de agua y de nitrógeno en el suelo.

Será necesaria la implementación de una política urgente de adaptación ante estos cambios. De estos datos se deduce que la evolución futura de los efectos que la acción antrópica está produciendo sobre el ciclo global del nitrógeno en los ecosistemas depende del posicionamiento político ambiental y de la evolución económica de cada país o región a escala global y de cada cuenca hidrográfica a escala local.

\section{Referencias}

ACA 2015. Resultados analíticos. Programa de seguimiento y control. Agencia Catalana del Agua. http://aca-web.gencat.cat/sdim2/inici.do

Almasri, M.N. 2007. Nitrate contamination of groundwater: a conceptual management framework. Environmental Impact Assessment Review 27: 220-242

Álvarez-Cobelas, M., Angeler, D.G., Sánchez-Carrillo, S. 2008. Export of nitrogen from catchments: a worldwide analysis. Environmental Pollution 156: 261-269

Arnell, N. 1998. Climate change and water resources in Britain. Climatic Change 39: 83-110

Belanger, T.V., Mikutel, D.F., Churchill, P.A. 1985. Groundwater seepage nutrient loading in a Florida lake. Water Resources 19: 773-781

Bouwman, A.F., Beusen, A.H.W., Billen, G. 2009. Human alteration of the global nitrogen and phosphorus soil balances for the period 1970-2050. Global Biochemical Cycles 23: 1-16

Camargo, J.A., Alonso, A. 2007. Contaminación por nitrógeno inorgánico en los ecosistemas acuáticos: problemas medioambientales, criterios de calidad del agua e implicaciones del cambio climático. Ecosistemas 16(2):98-110 
CHD 2015. Resultados analíticos completos 2006-2015. Confederación Hidrográfica del Duero, Valladolid, España. http://www.chduero.es/Inicio/ Gesti\%C3\%B3ndelaCuenca/Estadoycalidaddelasaguas/AguasSubterr\%C3\%A1neas/RedOficialdeControldeIEstadoQu\%C3\%ADmico/tabi d/568/Default.aspx

CHE 2016. Informe sobre la determinación de las aguas afectadas o en riesgo de contaminación por nitratos de origen agrario en la demarcación del Ebro (periodo 2012-2015). Confederación Hidrográfica del Ebro. Zaragoza, España.

Chenoweth, J., Hadjikakon, M., Zoumides, C. 2014. Quantifying the human impact on wáter resources: a critial review of the wáter footprint concept. Hydrology and Earth System Sciences 18: 2325-2342

Cherry, K.A., Shepherd, M., Withers, P.J.A., Mooney, S.J. 2008. Assessing the effectiveness of actions to mitigate nutrient loss from agriculture: $A$ review of methods. Science of the Total Environment 406: 1-23

CHG 2015. Plan Hidrológico 2016-2021. Confederación Hidrográfica del Guadiana. Ciudad Real, España. http://planhidrologico2015.chguadiana.es/?corp=planhidrologico2015\&url=61

CHGuad. 2015. Plan Hidrológico 2016-2021. Confederación Hidrográfica del Guadalquivir, Sevilla, España. http://www.chguadalquivir.es/demarcacion-hidrografica-guadalquivir

CHJ 2015. Programas de seguimiento del estado químico de las aguas subterráneas en la demarcación hidrográfica del Júcar. Informe anual 2015. Confederación Hidrográfica del Júcar. Valencia, España.

CHS 2016. Valoración del estado químico de las aguas subterráneas en la cuenca del Segura. Memoria técnica final. Periodo año hidrológico 2014-2015. Trabajos de campo de toma de muestras y análisis de la red integrada de control de calidad de aguas subterráneas del Segura (RICCASS). Expediente 03.0005.14.028. Confederación Hidrográfica del Segura. Murcia, España. https://www.chsegura.es/export/descargas/cuenca/redesdecontrol/calidadenaguassubterraneas/docsdescarga/Informe_estado_quimico_2014-2015.pdf

CHT 2015. Resultados analíticos campaña 2015. Confederación Hidrográfica del Tajo. Madrid, España. http://www.chtajo.es/Informacion\%20Ciudadano/Calidad_Vertidos/Resultados_Informes/Paginas/RISubControl Qu\%C3\%ADmico.aspx

Diaz, R.J., Rosenberg, R. 2008. Spreading dead zones and consequences for marine ecosystems. Science 321: 926-929

Díaz-Lezcano, M.I. 2007. Modelización de la contaminación difusa por nitratos en las aguas subterráneas de la cuenca del río Henares. Tesis Doctoral, Universidad Politécnica de Madrid, Madrid, España.

Domínguez, A., Tarjuelo, J.M., de Juan, J.A., Martínez, A. 2009. Efecto del cambio climático en los recursos hídricos y el regadío. En: Fundación General de Medio Ambiente de Castilla-La Mancha (ed). Impactos del cambio climático en Castilla-La Mancha, capítulo 4. Junta de CastillaLa Mancha, Toledo, España.

EEA 2016. Estudio sobre contaminación agrícola en acuíferos de la Subbética cordobesa 2016. Ecologistas en acción de Priego. https://www.ecologistasenaccion.org.

Eugercios, A.R. 2013. Interacciones acuífero-lago y biogeoquímica del nitrógeno en ambientes kársticos. Tesis Doctoral. Universidad Complutense de Madrid, Madrid, España. http://eprints.ucm.es/21579/

FAO 2011. The State of the world's land and wáter resources for food and agricultura (SOLAW) - Managing systems at risk. Food and Agriculture Organization of the United Nations, Rome and Earthscan, Londres, Reino Unido.

Galloway, J.N., Aber, J.D., Erisman, J.W., Seitzinger, S.P., Howarth, R.W. Cowling, E.B., Cosby, B.J. 2003. The nitrogen cascade. Bioscience 53 : 341-356

Galloway, J.N., Townsend, A.R., Erisman, J.W., Bekunda, M., Cai, Z., Freney, J.R., Martinelli, L.A., Seitzinger, S.P., Sutton, M.A. 2008. Transformation of the nitrogen cycle: recent trends, questions and potential solutions. Science 320: 889-892.

Gang, D., Qiang, Z., Zhang, Y., Kadari, R.K. 2005. Nonpoint sources. Water Environment Research 77: 2527-2575

Goderya, F.S., Dahab, M.F., Woldt, W.E., Bogardi, I. 1996. Incorporation of spatial variability in modeling non-point source groundwater nitrate polIution. Water Science and Technology 33: 233-40

Groffman, P.M., Butterbach-Bahl, K., Fulweiler, R.W., Gold, A.J., Morse, J.L., Stander, E.K., Tague, C., Tonitto, C., Vidon, P. 2009. Challenges to incorporating spatially and temporally explicit phenomena (hotspots and hot moments) in denitrification models. Biogeochemistry 93: 49-77
Gruber, N., Galloway, J.N. 2008. An Earth-system perspective of the global nitrogen cycle. Nature 451: 293-296

Harrison, J.A., Maranger, R.J., Alexander, R.B., Giblin, A.E., Jacinthe, P.A., Mayorga, E., Seitzinger, S.P., Sobota, D.J., Wollheim, W.M. 2008. The regional and global significance of nitrogen removal in lakes and reservoirs. Biogeochemistry 93: 143-157

Haygarth, P., Johnes, P., Butterfield, D., Foy, R., Withers, P. 2003. Land use for achieving 'good ecological status' of waterbodies in England and Wales: a theoretical exploration for nitrogen and phosphorus. Supplementary report for Defra project PE0203. Department for Environment Food and Rural Affairs. Chelmsford, Reino Unido.

Holden, J., Haygarth, P. M., MacDonald, J., Jenkins, A., Sapiets, A., Orr, H. G., Dunn, N., Harris, B., Pearson, P., McGonigle, D., Humble, A., Ross, M., Harris, J., Meacham, T., Benton, T. 2015. Agriculture's impacts on water quality. Global Food Security, 21pp. http://www.foodsecurity.ac.uk/ assets/pdfs/agriculture-water-quality-report.pdf

IPCC 2001. Cambio Climático: la base científica (resumen técnico). Aportación del Grupo de Trabajo I al Tercer Informe de Evaluación del Panel Intergubernamental de Cambio Climático. Cambridge University Press. Cambridge, Reino Unido.

IPCC 2007. Climate Change 2007 - The Physical Science Basis. Contribution of Working Group I to the Fourth Assessment Report of the IPCC. Cambridge University Press. Cambridge, Reino Unido.

IPCC 2013. Climate Change 2013 - The Physical Science Basis. Contribution of Working Group I to the Fifth Assessment Report of the IPCC Cambridge University Press. Cambridge, Reino Unido.

JDA 2015. Plan Hidrológico 2015-2021. Demarcaciones hidrográficas de Cuencas Mediterráneas Andaluzas, Guadalete y Barbate y Tinto, Odiel y Piedras. Junta de Andalucía. Sevilla, España. http://www.juntadeandalucia.es/medioambiente/site/portalweb/menuitem.220de8226575045b25f0 9a105510e1 ca/?vgnextoid=0bb66af68bb96310VgnVCM1000001325e50 aRCRD\&vgnextchannel=c418566029b96310VgnVCM2000000624e50a RCRD

Jeppesen, E., Kronvang, B., Olesen, J.E., Audet, J., Sondergaar, M., Hoffmann, C.C., Andersen, H.E., Lauridsen, T.L., Liboriussen, L., Larsen, S.E., Beklioglu, M., Meerhoff, M., Özen, A., Özkan, K. 2011. Climate change effects on nitrogen loading from cultivated catchments in Europe: implications for nitrogen retention, ecological state of lakes and adaptation. Hydrobiologia 663: 1-21

Kaushal, S.S., Groffman, P.M., Band, L.E., Elliott, E.M., Shields, C.A., Kendall, C. 2011. Tracking nonpoint source nitrogen pollution in humanimpacted watersheds. Environmental Science and Technology 45: 8225-8232

Kennedy, C.D., Genereux, D.P., Corbett, D.R., Mitasova, H. 2009. Relationships among groundwater age, denitrification and the coupled groundwater and nitrogen fluxes through a streambed. Water Resources Research 45: 1-15

Kim, O.A., Imhoff, J.F., Witzell, K.P., Junier, P. 2011. Distribution of denitrifying bacterial communitires in the stratified water columns and sediment-water interface in two freshwater lakes and the Baltic Sea. Aquatic Ecology 45: 99-112

Loáiciga, H.A., Maidment, D., Valdes, J.B. 2000. Climate change impacts in a regional karst aquifer, Texas, USA. Journal of Hydrology 227: 173-94

Martínez, J., Esteve, M.A., Guaita, N., Martínez-López, J. 2013. Modelización del efecto de los cambios de uso del suelo sobre los flujos de nutrientes en cuentas agrícolas costeras: el caso del Mar Menor (sudeste de España). Ecosistemas 22(3):84-94

O'Shea, L., Wade, A. 2009. Controlling nitrate pollution: an integrated approach. Land Use Policy 26: 799-808

OECC 2006. Plan Nacional de Adaptación al Cambio Climático: marco para la coordinación entre administraciones públicas para las actividades de evaluación de impoactos, vulnerabilidad y adaptación al Cambio Climático. Secretaría General para la Prevención de la Contaminación y del Cambio Climático, Ministerio del Medioambiente. Madrid, España. http://www.magrama.gob.es/es/

Olesen, J.E., Carter, T.R., Díaz-Amrona, C.H., Fronzek, S., Heidmann, T., Hickler, T., Holt, T., Minguez, M.I., Morales, P., Palutikov, J., Quemada, M., Ruiz-Ramos, M., Rubaek, G., Sau, F., Smith, B., Sykes, M. 2007. Uncertainties in projected impacts of climate change on European agriculture and ecosystems based on scenarios from regional cimate models. Climate Change 81: 123-143 
Ottosen, L.D.M., Risgaard-Petersen, N., Nielsen, L.P. 1999. Direct and indirect measurements of nitrification and denitrification in the rhizosphere of aquatic macrophytes. Aquatic Microbial Ecology 19, 81-91

PAC 2013. La Política Agraria Común (PAC) y la agricultura europea. Comisión Europea. http://www.mapama.gob.es/es/politica-agricola-comun/ cronograma-reforma-pac/

Patil, R., Olesen, J.E., Legdsmand, M., Porter, J.R. 2010. Effect of soil warming and rainfall patterns on soil $n$ cycling in Nothern Europe. Agriculture, Ecosystems and Environment 139: 195-205

PHIB 2015. Memoría del Plan Hidrológico de las Islas Baleares. Gobierno de las Islas Baleares. Consejería de Agricultura, Medio Ambiente y Territorio. Palma de Mallorca, Mallorca.

Rabalais, N.N. 2002. Nitrogen in aquatic ecosystems. Ambio: A Journal of the Human Environment 31: 102-112

Schindler, D.W., Dillon, P.J., Schreier, H. 2006. A review of anthropogenic sources of nitrogen and their effects on Canadian aquatic ecosystems. Biogeochemistry 79: 25-44

Schlesinger, W.H. 2009. On the fate of anthropogenic nitrogen. Proceedings of National Academy of Sciences, PNAS 106: 203-208

Seitzinger, S., Harrison, J.A., Böhlke, J.K., Bouwman, A.F., Lowrance, R. Peterson, B., Tobias, C., Van Drecht, G. 2006. Denitrification across landscapes and waterscapes: a synthesis. Ecological Applications 16: 2064-2090

Seitzinger, S.P., Mayorga, E., Bouwman, A.F., Kroeze, C., Beusen, A.H.W., Billen, G., Van Drecht, G., Dumont, E., Fekete, B.M., Garnier, J., Harrison, J.A. 2010. Global river nutrient export: a scenario analysis of past and future trends. Global Biogeochemical Cycles 24: 1-16

Shamrukh, M., Corapcioglu, M., Hassona, F. 2001. Modeling the effect of chemical fertilizers on ground water quality in the Nile Valley Aquifer, Egypt. Ground Water 39: 59-67
Soana, E., Racchetti, E., Laini, A., Bartoli, M., Viaroli, P. 2011. Soil budget, net export and potential sinks of nitrogen in the Lower Oglio river watershed (northern Italy). Soil Air Water 39: 956-965

Strebel, O., Duynisveld, W.H.M., Böttcher, J. 1989. Nitrate pollution in western Europe. Agriculture, Ecosystems and Environment 26: 189-214

Townsend, A.R., Howarth, R.W., Bazzaz, F.A., Booth, R.S., Clevelands, C.C., Collinge, S.K., Dobson, A.P., Epstein, P.R., Holland, E.A., Keeney, D.R., Mallin, M.A., Rogers, C.A., Wayne, P., Wolfe, A.H. 2003. Human health effects of a changing global nitrogen cycle. Frontiers in Ecology and the Environment 1: 240-246

Turner, J., Albrechtsen, H.J., Bonell, M., Duguet, J.P., Harris, B., Meckenstock, R., McGuire, K., Moussa, R., Peters, N., Richnow, H.H., Sherwood-Lollar B., Uhlenbrook, S., Lanen, H. 2006. Future trends in transport and fate of diffuse contaminants in catchments, with special emphasis on stable isotope applications. Hydrological Processses 20: 205-213

Varela, M. 1991. Situación de la contaminación por nitratos en las aguas subterráneas del territorio peninsular y balear. Informaciones y estudios del servicio geológico del MOPT 53: 1-70

Welch, H., Green, C., Coupe, R.H. 2011. The fate and transport of nitrate in shallow groundwater in northwestern Mississippi, USA. Hydrogeology Journal 19: 1239-1252

Winter, T.C. 1999. Relation of streams, lakes and wetlands to groundwater flow systems. Hydrogeology Journal 7: 28-45

Winter, T.C., Buso, D.C., Shattuck, P.C., Harte, P.T., Vroblesky, D.A., Goode, D.J. 2008. The effect of terrace geology on ground-water movement and on the interaction of ground water and surface water on a mountainside near Mirror Lake, New Hampshire, USA. Hydrological Processes 22: 21-32

Xepapadeas, A. 2011. The economics of non-point source pollution. Annual Review of Resource Economics 3: 355-373 